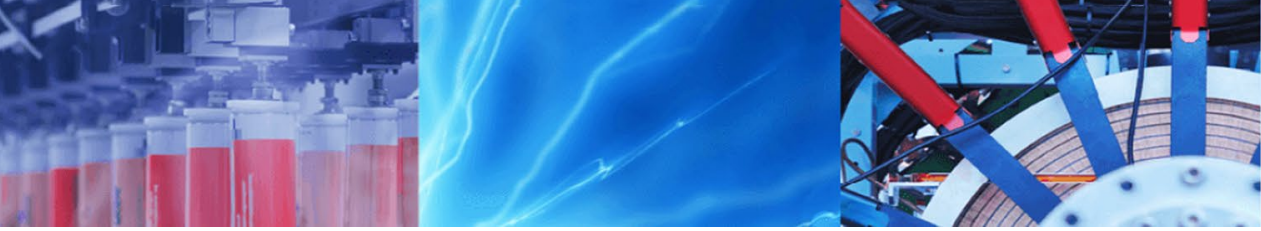

Research Article

\title{
Preparation of high-concentration colloid solutions of metallic copper particles and their use in metal-metal bonding processes
}

\author{
Yuki Suga $^{1} \cdot$ Kouichi Nakashima $^{1} \cdot$ Noriko Yamauchi $^{1} \cdot$ Yusuke Yasuda $^{2} \cdot$ Toshiaki Morita $^{2} \cdot$ Yoshio Kobayashi $^{1}$ (i)
}

(c) Springer Nature Switzerland AG 2019

\begin{abstract}
A high-concentration colloid solution of metallic copper nanoparticles was prepared by the reduction of copper ions in aqueous solutions, and the nanoparticles were used to study metal-metal bonding. The colloid solution of metallic copper nanoparticles was prepared by mixing an aqueous solution of $\mathrm{CuCl}_{2}$ and an aqueous solution of hydrazine (reductant) in the presence of citric acid and n-hexadecyltrimethylammonium bromide (stabilizers) at a final copper concentration as high as $0.1 \mathrm{M}$. The copper-particle size was ca. submicrometer. Many tiny particles with sizes of ca. 10-20 nm were deposited on the submicron-sized particles at high hydrazine concentrations. The tiny particles were considered to be produced through the growth of particle nuclei generated at the final reaction stage by residual hydrazine derived from the high hydrazine concentration. Metallic-copper discs were successfully bonded by annealing at $400{ }^{\circ} \mathrm{C}$ and a pressure of 1.2 MPa for $5 \mathrm{~min}$ in hydrogen gas with the help of the metallic-copper particles. The shear strength of the bonded copper discs was then measured. The highest shear strength of 41.7 MPa was recorded for the tiny particles/ immobilized particles, which could be explained by the low melting points of the tiny particles and the increase in bulk density provided by the dominant incorporation of the tiny particles into voids between the submicron-sized particles.
\end{abstract}

Keywords Copper $\cdot$ Nanoparticle $\cdot$ Aqueous solution $\cdot$ Metal-metal bonding $\cdot$ Filler

\section{Introduction}

Metal-metal bonding is an indispensable process especially in fields such as electronics and metal processing [1-4]. Conventional metal-metal bonding is performed by annealing two or more metallic materials at high temperatures [5-8]. During the bonding, the components of one metal diffuse into the other metal, which bonds the metals. The high-temperature annealing promotes the diffusion. Because the high annealing temperature may damage the metals to be bonded, the annealing temperature should be lowered. The bonding can be performed at low temperatures by using a metallic solder or a metallic filler with a low melting point [9]. Tin-lead alloy, which has a low melting point, is a representative filler used in metal-metal bonding [10-12]. However, if the bonded metals are exposed to temperatures higher than their melting point, they may be released due to re-melting of the alloy. The tin-lead alloy also faces a problem with its toxicity. As several researchers have suggested, its use is limited for the sake of environmental protection and human health concerns, [13-17]. Various lead-free, tin-related alloys also have low melting points. Considering health and environmental concerns, metal-metal bonding processes using lead-free, tin-related alloys have been proposed. However, such alloys still face the same problem derived from their low melting points that the tin-lead alloy does.

Particles of metals such as metallic nanoparticles and metallic submicron-sized particles can contact other materials efficiently and effectively achieve metal-metal

$\triangle$ Noriko Yamauchi, noriko.yamauchi.12@vc.ibaraki.ac.jp; $\bigotimes$ Yoshio Kobayashi, yoshio.kobayashi.yk@vc.ibaraki.ac.jp| ${ }^{1}$ Department of Materials Science and Engineering, Graduate School of Science and Engineering, Ibaraki University, 4-12-1 Naka-narusawa-cho, Hitachi, Ibaraki 316-8511, Japan. ${ }^{2}$ Hitachi Research Laboratory, Hitachi Ltd., 7-1-1 Omika-cho, Hitachi, Ibaraki 319-1292, Japan.

SN Applied Sciences (2019) 1:925 | https://doi.org/10.1007/s42452-019-0845-1 
bonding, since they have a larger apparent surface area than that of their bulk. In particular, nanoparticles have melting points lower than those of their bulk of metals [18-20]. The lower melting points can be applied to metal-metal bonding at low temperatures.

Since metallic silver is electrically conductive, thermally conductive and chemically stable, its particles are good candidates as a nanoparticle filler. Several researchers have studied the metal-metal bonding process using silver-based metallic particles as filler [21-29], and these studies indicate that the silver-based particles exert excellent metal-metal bonding properties. However, the cost of metallic silver is higher than that of many other metals that are expected to function as a metallic particle filler. In addition, electrochemical migration takes place for metallic silver [30-32], which may deteriorate the bonding performed with metallic-silver nanoparticles as a filler.

Metallic copper also has excellent electrical and thermal conductivities and is available at a low cost provided the method for producing metallic copper particles is simple and the obtained particles are chemically stable. In addition, the electrochemical migration of copper does not occur as easily as that of silver, as several researchers have suggested [33,34]. Accordingly, metallic copper particles are another candidate as a filler for bonding. Metal-metal bonding using metallic copper particles has been extensively studied by several researchers $[25$, 35-39]. In most of their works, the metallic copper particles have been produced in organic solvents, which are unsafe and impart a high environmental load. Apart from their works, several methods for fabricating metallic copper particles have been also proposed. Krasovskii et al. [40] proposed a method for producing metallic copper particles by a technique using a DC thermal plasma, and studied on thermal evolution of nonmetallic impurities and surface passivation of the particles. Mousavi-Kamazani et al. [41] produced nanoparticles composed of metallic copper and cuprous oxide by annealing copper complex in argon, and investigated their photocatalytic activity. Although the two methods worked well, they appeared to be complicated in operation and to require many steps compared to the methods utilizing chemical reaction in liquid phase, respectively. Methods using aqueous solution have been reported by several researchers [42-47]. In the methods, copper ions are reduced with reductants in aqueous solution. The methods are quite simple, safe, and do not impart a high environmental load. From this viewpoint, our research group has produced metallic copper particles produced in aqueous solution, and studied metal-metal bonding using the particles [48-56].

Particles tend to aggregate at large ionic strength. If the aggregation occurs for the metallic copper particles, the particles no longer maintain their excellent bonding ability because of the increase in their size. Therefore, in our previous work, colloid solutions of metallic copper particles have been prepared at low concentrations to prevent the species from aggregating: the copper concentration in the as-prepared particle colloid solutions, prepared by reducing copper ions with hydrazine in an aqueous medium, was as low as $10^{-2} \mathrm{M}[48-51,54-56]$. The mass production of fillers is required for their practical use. Therefore, it is necessary to synthesize the colloid solution at high concentrations of raw chemicals, simplify the preparation method, and reduce the cost. The present work prepared high-concentration colloid solutions of metallic Cu particles at high copper concentrations. The metal-metal bonding properties of the particles were also studied. Particle morphology, such as particle size and particle shape, which may also be related to particle aggregation, should be dependent on parameters such as the reaction time and concentrations of chemicals, and they should influence the metal-metal bonding properties. Such influence was investigated in the present work.

\section{Experimental}

\subsection{Chemicals}

Copper acetate monohydrate $\left(\left(\mathrm{CH}_{3} \mathrm{COO}\right)_{2} \mathrm{Cu} \cdot \mathrm{H}_{2} \mathrm{O}\right)(>99 \%)$ and hydrazine monohydrate $(>98.0 \%)$ were used as the source of metallic-copper particles and reducing reagent, respectively. The chemicals used for stabilizing the particle colloid solutions were citric-acid monohydrate (>99.5\%) and cetyltrimethylammonium bromide (CTAB) (99\%). All chemicals were purchased from Kanto Chemical Co., Inc. (Tokyo, Japan) and used as received. All preparations were performed in ion-exchanged water distilled with an Advantec RFD372FC water distillation apparatus. The water was deaerated by bubbling with nitrogen gas for 30 min prior to the preparation of various aqueous solutions.

\subsection{Preparation}

Metallic-copper particles were fabricated by reducing copper ions with hydrazine in aqueous solution and explained as follows. A freshly prepared hydrazine aqueous solution was added to an aqueous solution of CTAB, citric acid, and $\left(\mathrm{CH}_{3} \mathrm{COO}\right)_{2} \mathrm{Cu} \cdot \mathrm{H}_{2} \mathrm{O}$ under vigorous stirring at a reduction temperature of $25^{\circ} \mathrm{C}$. The initial concentrations of these chemicals were $0.1 \mathrm{M}\left(\mathrm{CH}_{3} \mathrm{COO}\right)_{2} \mathrm{Cu}, 1-6 \mathrm{M}$ hydrazine, $5.0 \times 10^{-4}-5.0 \times 10^{-3} \mathrm{M}$ citric acid and $5.0 \times 10^{-2} \mathrm{M} \mathrm{CTAB}$. The reduction time was $24-92 \mathrm{~h}$. After the reduction, the obtained particles were washed by the following procedure (repeated three times): centrifugation at 10,000 rpm, 
removal of the supernatant, the addition of water and shaking of the mixture with a vortex mixer to disperse the particles.

\subsection{Characterization}

The particles were characterized by transmission electron microscopy (TEM) and X-ray diffractometry (XRD). TEM electrograms were taken with a JEOL JEM-2100 microscope operating at $200 \mathrm{kV}$. The TEM samples were prepared by dropping and evaporating the particle colloid solution on a collodion-coated copper grid. The number-averaged particle size and standard deviation of the particle-size distribution were determined by measuring multiple particle diameters in the TEM images. The XRD patterns of copper particles were acquired with a Rigaku Ultima IV X-ray diffractometer at $40 \mathrm{kV}$ and $30 \mathrm{~mA}$ with $\mathrm{Cu} \mathrm{Ka}$ radiation. To prepare the powder samples for the XRD measurement, the particles from the final removal of supernatant were dried at room temperature for $24 \mathrm{~h}$ under vacuum. The crystal size was estimated by using the Scherrer equation, as follows.

$D=\frac{K \lambda}{\beta \cos \theta}$,

where $D$ is the crystal size, $\lambda$ is the $\mathrm{X}$-ray wavelength $(1.5406 \AA$ ),$\beta$ (expressed in radians) is the full width at half maximum, $\theta$ is the Bragg angle, and $K$ is the Scherrer constant (0.9).

The metal-metal bonding property of the particle powder was investigated by the same setup as used in our previous works [24,57-59] and is explained as follows. The sample for investigation of metal-metal bonding property of the particle powder was made by hand, as follows. The same powder sample as that obtained for the XRD measurement was spread on a metallic-copper disc as a stage (with a diameter of $10 \mathrm{~mm}$ and a thickness of $5 \mathrm{~mm}$ ). Then, a metallic-copper disc as a plate (with a diameter of $5 \mathrm{~mm}$ and a thickness of $2.5 \mathrm{~mm}$ ) was put on top of the powder sample. The metallic copper discs sandwiching the powder sample were compressed at 1.2 MPa under annealing in hydrogen at $400^{\circ} \mathrm{C}$ for $5 \mathrm{~min}$ with a Shinko Seiki vacuum reflow system. The bonding property of the particles was evaluated in terms of the shear strength required to separate the bonded disc and stage. The shear strength was measured with a Seishin SS-100KP bond tester at a shear rate of $30 \mathrm{~mm} / \mathrm{min}$. The bonding and the measurement of shear strength were performed two or three times for each sample. The average of measured shear strengths was regarded as bonding strength of particles. The disc surface was observed by scanning electron microscopy (SEM), namely, a JEOL JSM-5600 LV, after the shear strength was measured.

\section{Results and discussion}

\subsection{Morphology of particles}

\subsubsection{Effect of reduction time}

The color of the solution turned dark red or brown gradually after the addition of hydrazine. Figure 1 shows photographs of colloid solution prepared at various reduction times. There was no large difference in the strength of color among the reduction times examined, which implied that the reaction nearly complete at $24 \mathrm{~h}$. The colors of all the solutions appeared to contain a tint of red. Since
Fig. 1 Photographs of colloid solutions prepared at reduction times of a 24 , b 48, c 72 , and $\mathbf{d} 92 \mathrm{~h}$. The concentrations of $\left(\mathrm{CH}_{3} \mathrm{COO}\right)_{2} \mathrm{Cu}$, hydrazine, citric acid, and CTAB were 0.1 , $6.0,5.0 \times 10^{-3}$, and $5.0 \times 10^{-2}$, respectively
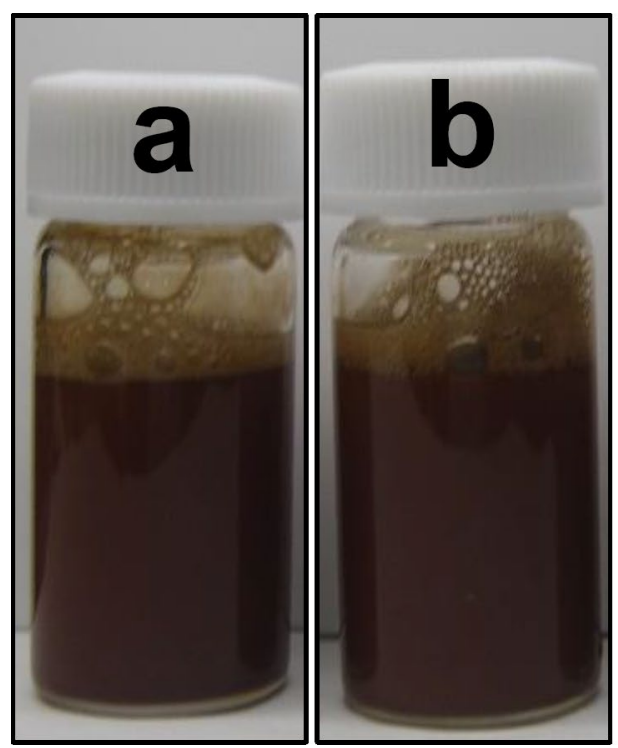

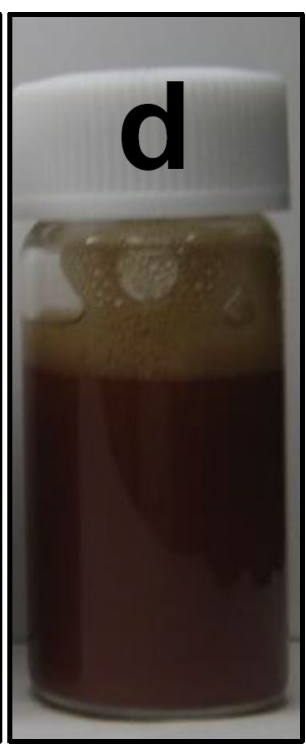

SN Applied Sciences A SPRINGER NATURE journa 
the surface plasmon resonance absorption of metallic $\mathrm{Cu}$ particles resulted in a red color, the color change implied that metallic Cu particles were already produced at $24 \mathrm{~h}$. Figure 2 shows TEM images of the particles. The particles had rough surfaces. Almost all of the particles examined had a size in the range of ca. $50-150 \mathrm{~nm}$. The average particle sizes were $92.2 \pm 60.7,92.6 \pm 29.9,94.7 \pm 30.9$ and $81.4 \pm 28.7 \mathrm{~nm}$ for reduction times of $24,48,72$ and $92 \mathrm{~h}$, respectively. The particles for the different reduction times were synthesized in different reaction vessels. Accordingly, we considered that there was no large difference in particles size among the reduction times within the statistical error.

\subsubsection{Effect of citric acid concentration}

Figure 3 shows photographs of colloid solutions prepared at various citric acid concentrations. For all the citric acid concentrations examined, the color of the solution turned dark red or brown gradually after the addition of hydrazine. Accordingly, the production of metallic Cu particles was implied for the preparation in the range of citric acid concentrations examined. Figure 4 shows TEM images of the particles. The average particle sizes were $122.4 \pm 54.8,86.3 \pm 37.3,91.6 \pm 72.7$, $84.8 \pm 34.2$ and $92.6 \pm 45.1 \mathrm{~nm}$ for citric acid concentrations of $5.0 \times 10^{-4}, 1.3 \times 10^{-3}, 2.5 \times 10^{-3}, 3.8 \times 10^{-3}$ and $5.0 \times 10^{-3} \mathrm{M}$, respectively. For a concentration as low as $5.0 \times 10^{-4} \mathrm{M}$, many particles larger than $200 \mathrm{~nm}$ were also clearly observed. The particle size decreased to $86.3 \pm 37.3 \mathrm{~nm}$ with increasing concentrations up to $1.3 \times 10^{-3} \mathrm{M}$, because citric acid was considered to be adsorbed on the particles and then to control further deposition of generated nuclei on the particles followed by the growth of the particles in the concentration range of $5.0 \times 10^{-4}-1.3 \times 10^{-3} \mathrm{M}$. The particles were colloidally stabilized with citric acid, which controlled the growth of the particles. There was no large difference in particle size among concentrations over $5.0 \times 10^{-4} \mathrm{M}$. The increase in citric acid concentration led to an increase in the ionic strength of the solution. Since increases in the ionic strength compress the double layer on solid materials, such as colloidal particles [60-62], the double-layer repulsion between Cu particles was probably reduced at
Fig. 2 TEM images of particles. Samples a-d were obtained from the colloid solutions a-d shown in Fig. 1
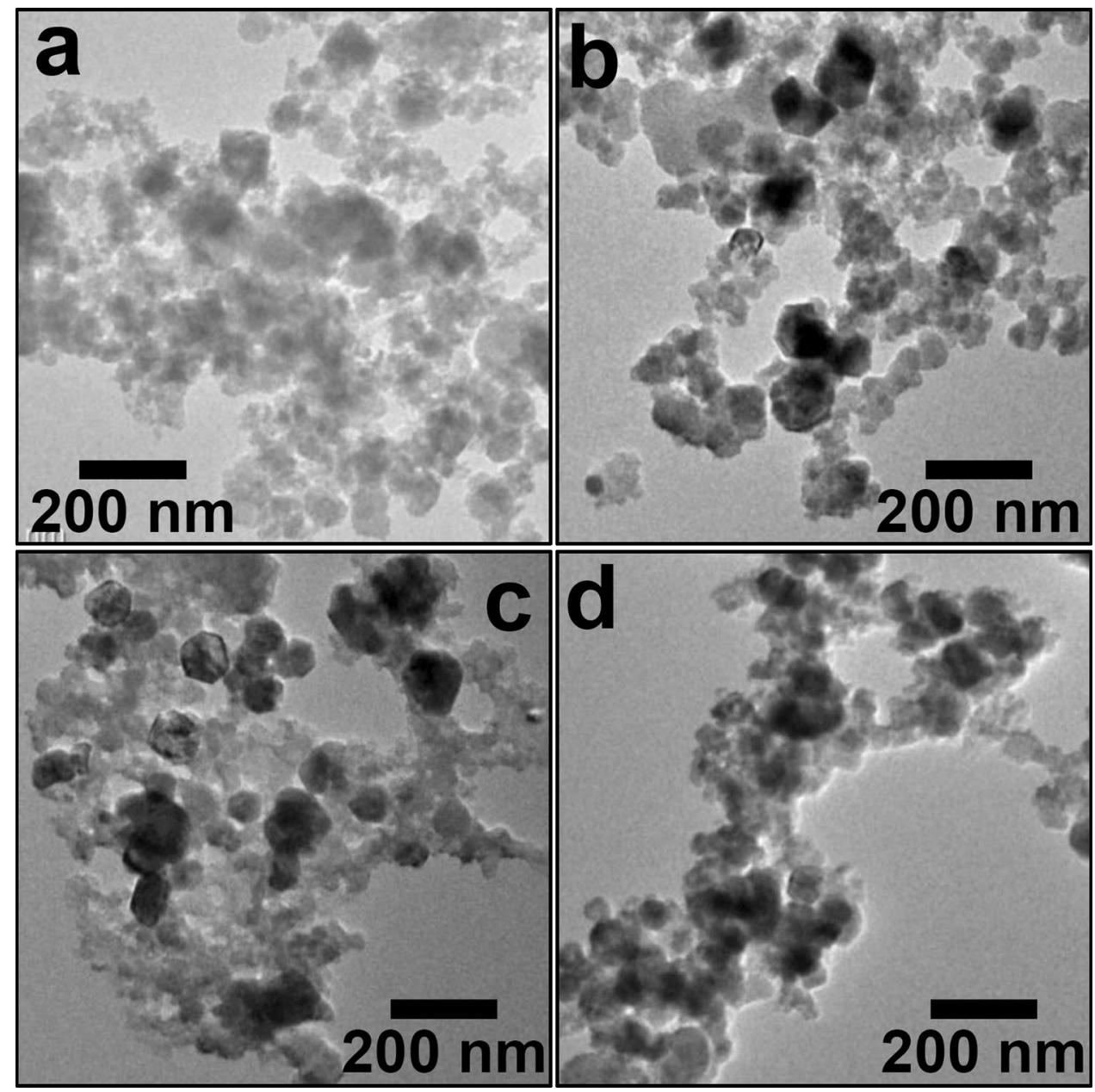

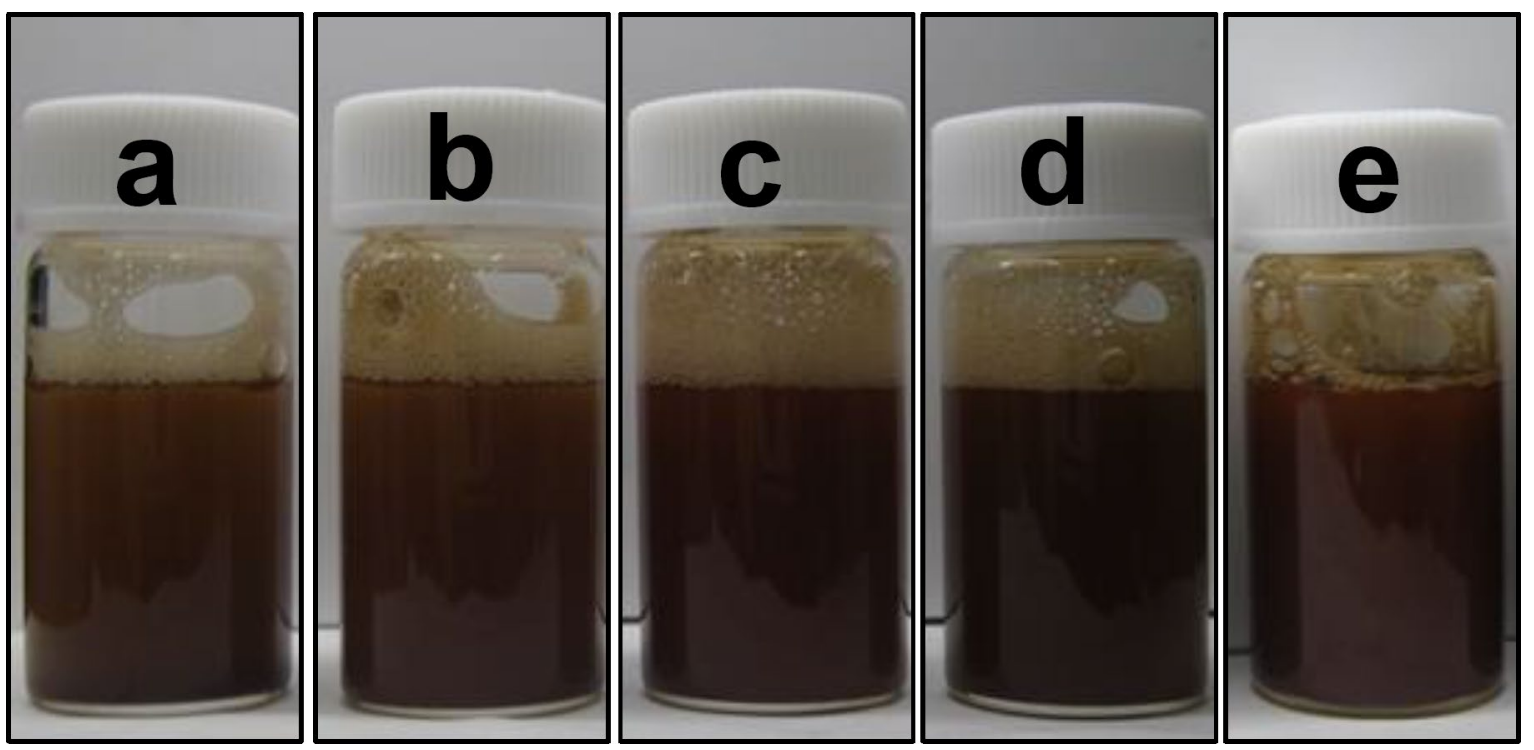

Fig. 3 Photographs of colloid solutions prepared at citric acid concentrations of a $5.0 \times 10^{-4}, \mathbf{b} 1.3 \times 10^{-3}, \mathbf{c} 2.5 \times 10^{-3}$, d $3.8 \times 10^{-3}$, and e $5.0 \times 10^{-3} \mathrm{M}$. The concentrations of $\left(\mathrm{CH}_{3} \mathrm{COO}\right)_{2} \mathrm{Cu}$, hydrazine, and CTAB were $0.1,6.0$, and $5.0 \times 10^{-2}$, respectively. The reduction time was $24 \mathrm{~h}$

Fig. 4 TEM images of particles. Samples a-e were obtained from the colloid solutions a-e shown in Fig. 3

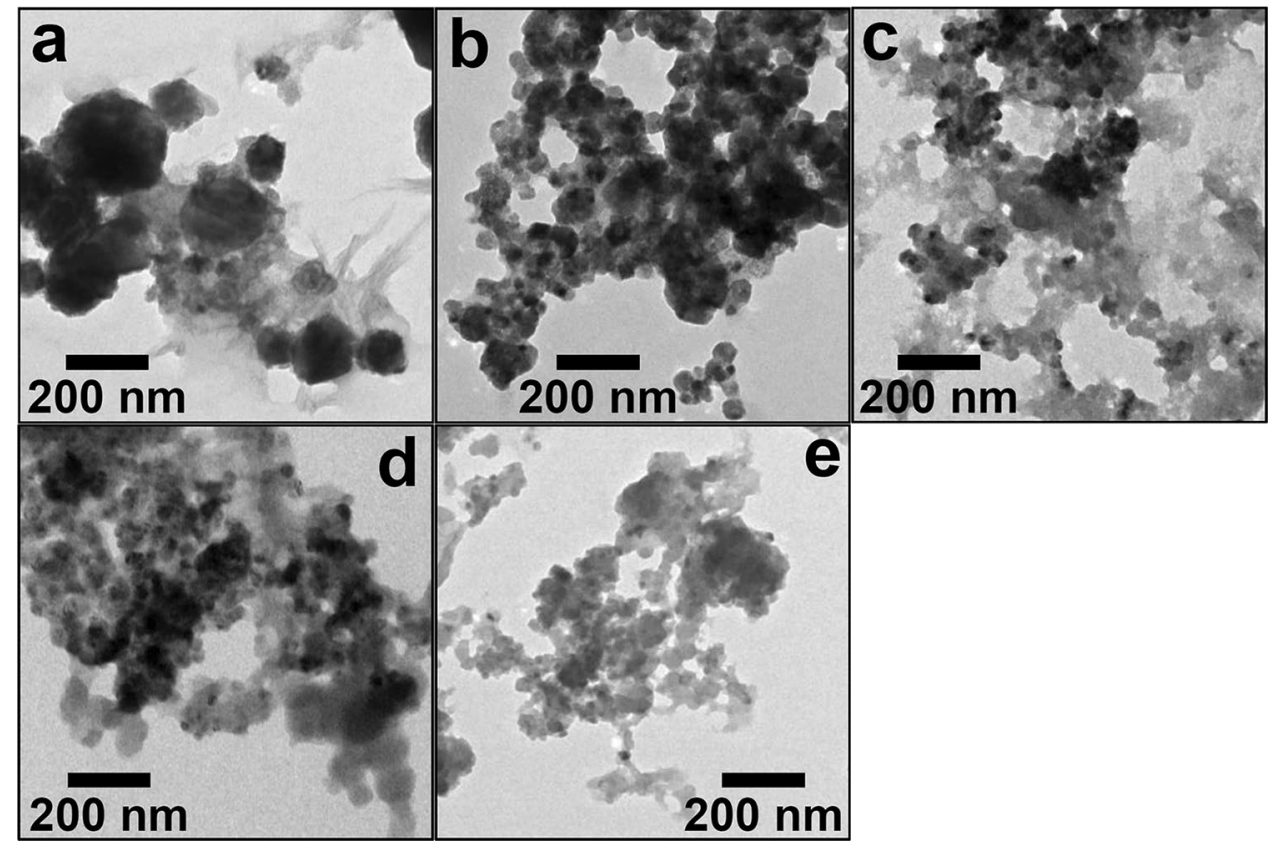

the high citric acid concentrations. The increased ionic strength would favor Cu nuclei aggregation. Thus, at the high citric acid concentrations, the colloidal stabilization of Cu particles based on the surface adsorption of citric acid and the increase in ionic strength provided by the addition of citric acid probably took place at the same time, or the effect of the increase in ionic strength offset the colloidal stabilization. Consequently, the offset provided no large difference in particle sizes.

\subsubsection{Effect of hydrazine concentration}

Figure 5 shows photographs of colloid solutions prepared at various hydrazine concentrations. Prior to the addition of hydrazine, the color of the solution was blue, which was attributed to $\mathrm{Cu}^{2+}$. For all the hydrazine concentrations examined, the color of the solution turned dark red or brown gradually after the addition of hydrazine, as shown in Fig. 5. Accordingly, the production of metallic 

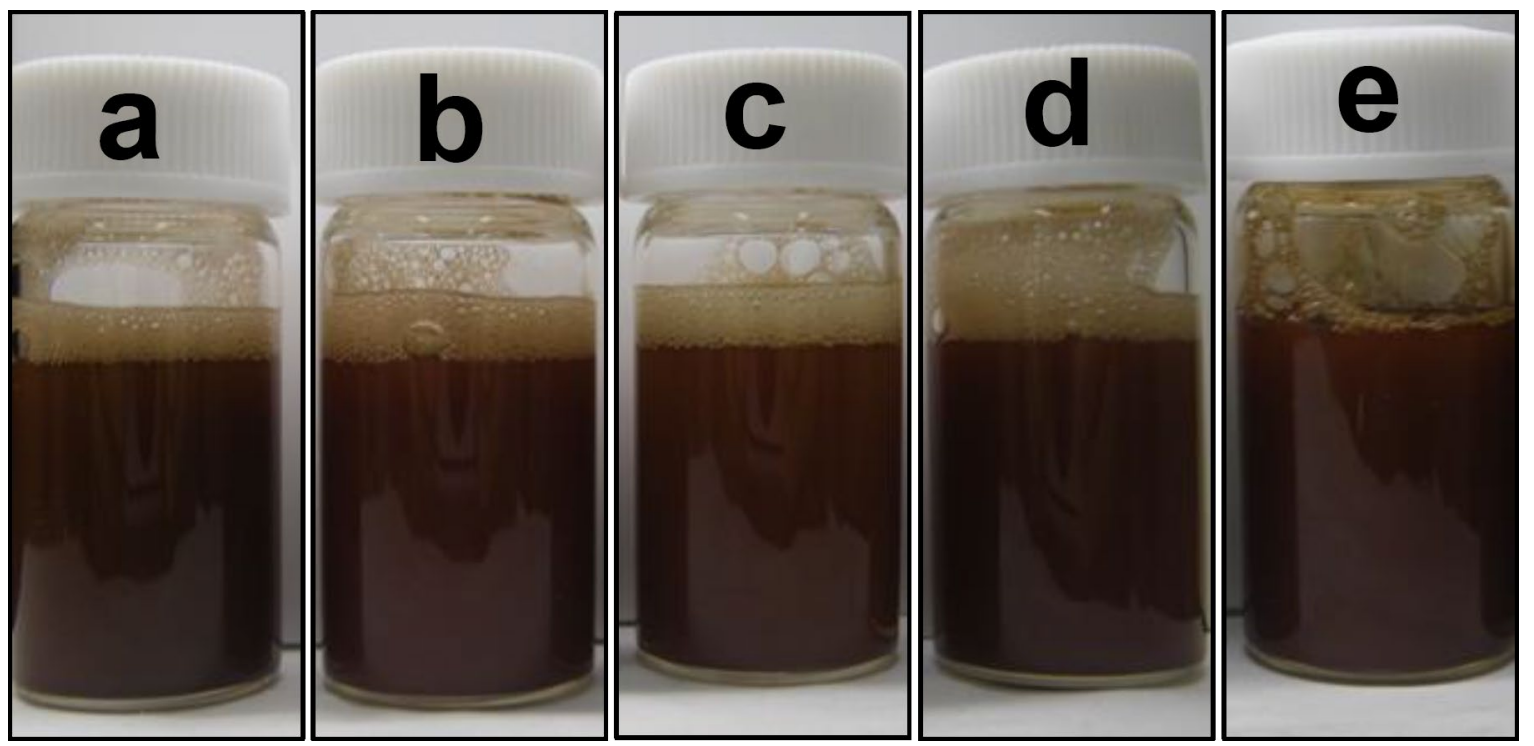

Fig. 5 Photographs of colloid solutions prepared at hydrazine concentrations of $\mathbf{a} 0.60, \mathbf{b} 1.5, \mathbf{c} 3.0, \mathbf{d} 4.5$, and e $6.0 \mathrm{M}$. The concentrations of $\left(\mathrm{CH}_{3} \mathrm{COO}\right)_{2} \mathrm{Cu}$, citric acid, and CTAB were $0.1,5.0 \times 10^{-3}$, and $5.0 \times 10^{-2}$, respectively. The reduction time was $24 \mathrm{~h}$

Cu particles was implied for the preparation in the range of hydrazine concentrations examined. There was no large difference in color tone among the hydrazine concentrations. Accordingly, the hydrazine concentration as small as $0.6 \mathrm{M}$ was considered to be enough for the reduction. Figure 6 shows TEM images of the particles. For all the hydrazine concentrations examined, the particle sizes appeared to be widely distributed in a range from tens of nanometers to submicrometer. The average particle sizes were $163.9 \pm 99.6,107.6 \pm 54.3,135.1 \pm 65.3,136.8 \pm 45.9$ and $111.9 \pm 51.4 \mathrm{~nm}$ for hydrazine concentrations of 0.6 , $1.5,3.0,4.5$ and $6.0 \mathrm{M}$, respectively. No strong dependence of particle morphology on the hydrazine concentration was seen in the TEM observation. Figure 7 shows SEM images of the particles. It should be noted that many tiny particles with a size of ca. 10-20 nm were on the surfaces of the submicron-sized particles for hydrazine concentrations as high as 3.0-6.0 M, though such particles were not observed at hydrazine concentrations between 0.6 and 1.5 M. The reduction of $\mathrm{Cu}^{2+}$ to form particles continued
Fig. 6 TEM images of particles. Samples a-e were obtained from the colloid solutions a-e shown in Fig. 5

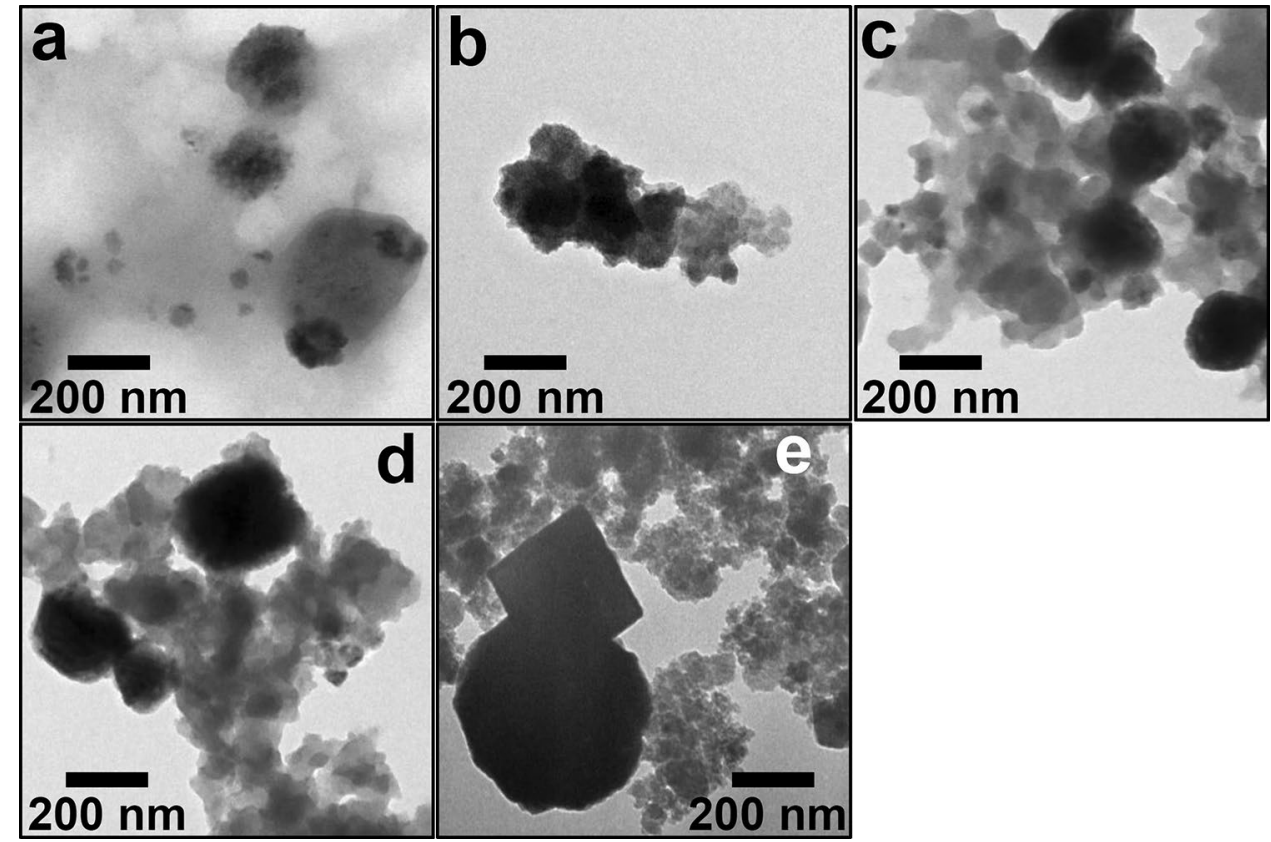


Fig. 7 SEM images of particles. Samples a-e were obtained from the colloid solutions a-e shown in Fig. 5

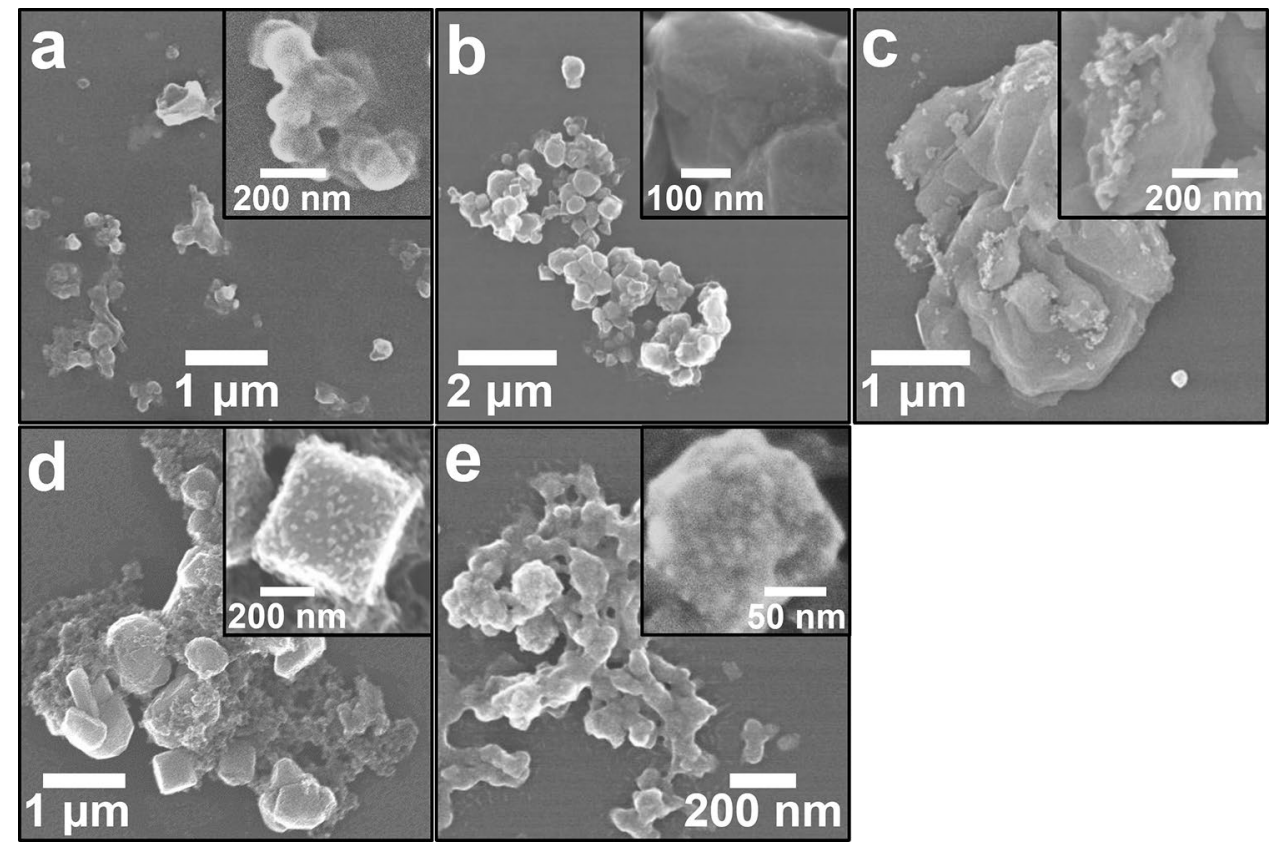

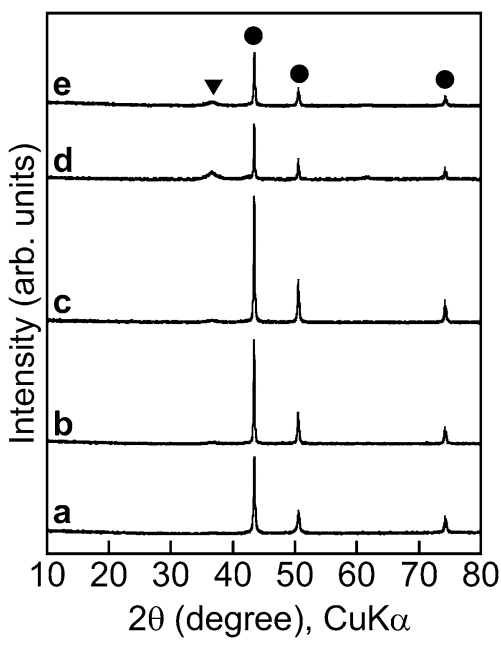

Fig. 8 SEM images of particles. The powders used were the same as those represented in Figs. 5 and 6. Filled circle: metallic copper, and filled inverted triangle: $\mathrm{Cu}_{2} \mathrm{O}$

at high hydrazine concentrations. First, the particle nuclei that were generated at an earlier reaction stage grew to form the submicron-sized particles. After the formation of submicron-sized particles, particle nuclei were also generated at the final reaction stage because the hydrazine remained at sufficiently high concentrations. The particle nuclei were considered to grow and form the tiny particles, which were deposited on the submicron-sized particles. Figure 8 shows XRD patterns of the particles. For all samples, three peaks were recorded at $43.3^{\circ}, 50.5^{\circ}$ and $74.2^{\circ}$, and the peaks were attributed to the (111), (200) and (220) planes of cubic copper, respectively (JCPDS card
No. 04-0836). For several samples, peaks assigned to $\mathrm{Cu}_{2} \mathrm{O}$ (JCPDS card No. 05-0667) were detected at $36.6^{\circ}$, though their intensities were weaker than those of cubic copper. The application of the Scherrer equation to the XRD line broadening of the peak at $43.3^{\circ}$ provided average metallic copper crystal sizes of $67.3,61.1,63.3,59.8$ and $61.1 \mathrm{~nm}$ for hydrazine concentrations of $0.6,1.5,3.0,4.5$ and $6.0 \mathrm{M}$, respectively. The crystal sizes at the hydrazine concentrations examined did not significantly differ, but they were smaller than the average particle sizes estimated by TEM. This result roughly indicated that the metallic copper particles were polycrystalline.

\subsection{Bonding property}

Photographs of the copper stages after the measurement of shear strength are shown in Fig. 9. The particles were fabricated at various hydrazine concentrations, or the same as in Figs. 6 and 7. For all the samples examined, brown products, which are clearly metallic copper, were observed on the stage. Surfaces that appeared to be the same as the copper stage were also observed in places. This observation indicated that the particle powder combined with the copper stage, which implied that the copper discs were strongly bonded. SEM images of the copper stages after the shear strength was measured are shown in Fig. 10. For all the samples, particles that appeared to be sintered were observed on the surface. These observations of sintering also implied strong bonding. For the hydrazine concentration of 3.0 M (Fig. 10c), many dimples were observed on the surface. The tearing of strongly bonded metals with 
Fig. 9 Photographs of copper disc stages after the measurement of shear strength. The powders used were the same as those represented in Figs. 5 and 6

Fig. 10 SEM images of the copper stages shown in Fig. 9
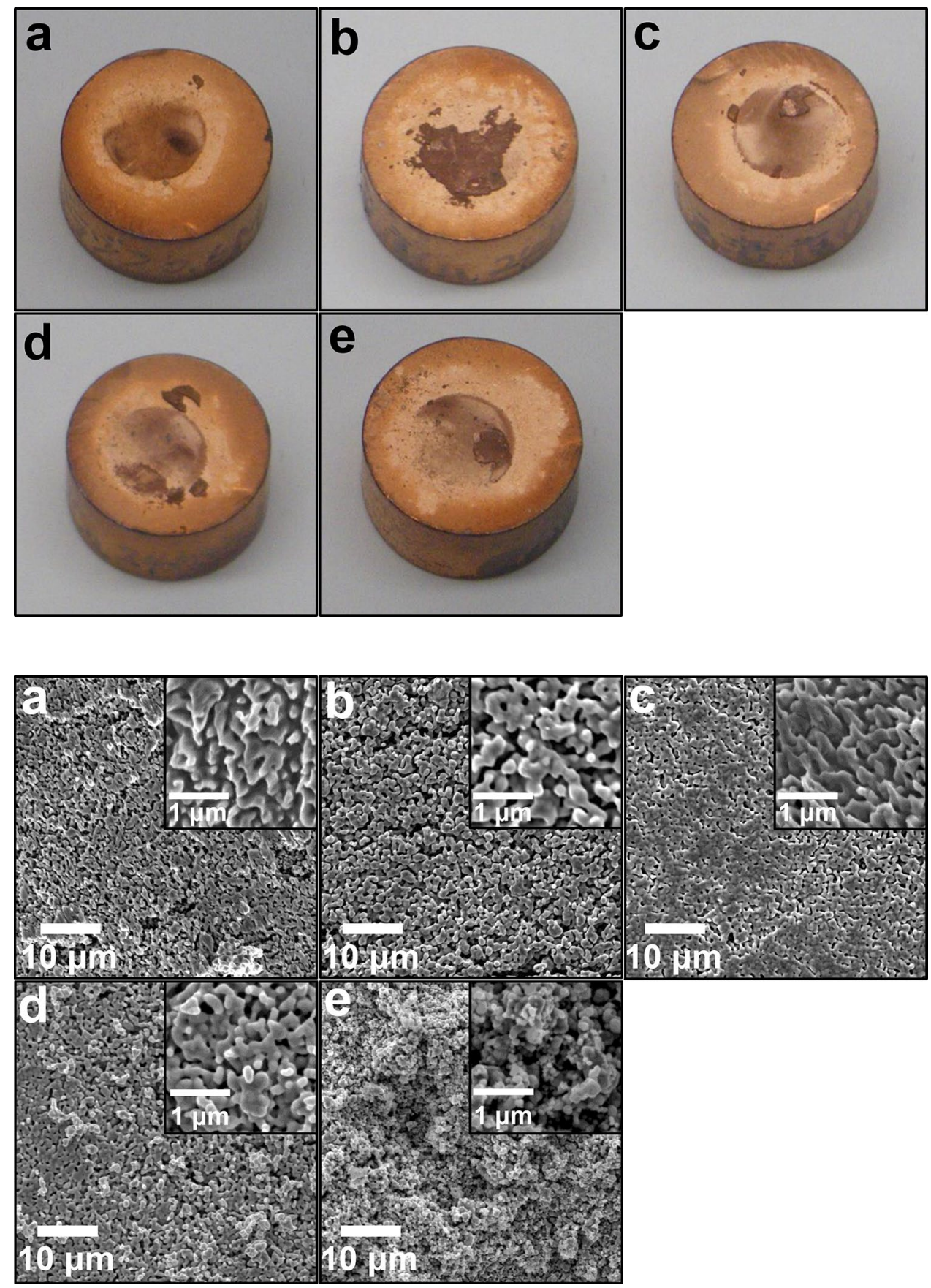

shearing stress forms the dimples in the bonded region [25]. This observation of dimples implied that the strongest bonding was obtained for the particles fabricated at the hydrazine concentration of 3.0 M. Dimples were also observed for the hydrazine concentration as low as $0.60 \mathrm{M}$, which implied the strong bonding. The shear strength is plotted as a function of hydrazine concentration in Fig. 11. Because the metallic copper discs sandwiching the powder sample were made by hand, their qualities should have been different somewhat. Accordingly, taking into account of the statistical error, the shear strength should be discussed. The shear strength was $19.3 \mathrm{MPa}$ at the hydrazine concentration of $0.6 \mathrm{M}$, which might have been enough high for the formation of dimples. The shear strength roughly tended to increase with increasing concentration from 0.6 to $3.0 \mathrm{M}$ within the statistical error. This tendency was also obtained in our previous works $[49,50,54,56]$, of which the associated mechanism is described as follows. A large amount of $\mathrm{Cu}$ oxide is contained in particles at low hydrazine concentrations. The removal of oxygen from the $\mathrm{Cu}$ oxide probably takes place during bonding. The oxygen

\section{SN Applied Sciences}




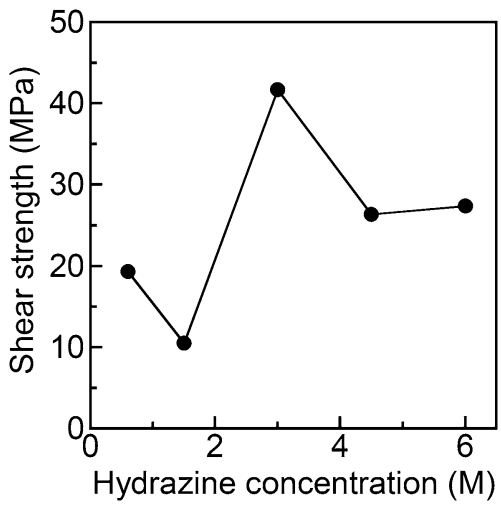

Fig. 11 Shear strength versus hydrazine concentration. The powders used were the same as those represented in Figs. 5 and 6

removal produces voids in the particles after bonding, which prevent the metallic copper from having efficient contact with other particles and the copper disc. As a result, the shear strength is small at low hydrazine concentrations. In the present work, Cu oxide was not remarkably detected in the XRD (Fig. 8). Accordingly, this mechanism was not considered to dominate in the present work. A shear strength as high as $41.7 \mathrm{MPa}$ at $3.0 \mathrm{M}$ was achieved, the strongest among our works on $\mathrm{Cu}-\mathrm{Cu}$ bonding [48-56]. This result supported the implication obtained by the SEM observation (Fig. 10c). Regarding the SEM observation of particles (Fig. 7c), many tiny particles were immobilized on large particles. The tiny particles might have had low melting points compared to those of the large particles because of the small particle sizes. Consequently, the bonding was considered to be promoted with the reduced melting points. In addition, the tiny particles could be dominantly incorporated into the voids formed between the large particles, which increased the density of the particle powder layers. The formation of dense layers provided efficient sintering that took place among the large particles and gave the strongest bonding. Over 3.0 $\mathrm{M}$, the shear strength tended to decrease from 41.7 to $27.4 \mathrm{MPa}$ with increasing concentration. This tendency was also obtained in our previous works $[49,50,54,56]$, for which the mechanism is described as follows. Unreacted hydrazine that remains as an impurity in the solution due to the high hydrazine concentration is adsorbed on the particle surface and incorporated into particles. Then, the unreacted hydrazine is removed from the particles via sublimation or evaporation during bonding, forming voids in the particles. The formation of voids spoils bonding. This mechanism was also considered for the present work. Accordingly, the effect of the formation of voids derived from the remaining hydrazine was considered to overcome the effect attributed to the formation of tiny particles at hydrazine concentrations over $3.0 \mathrm{M}$.

\section{Conclusions}

A method for preparing aqueous colloid solutions of metallic copper particles at a copper concentration as high as $0.1 \mathrm{M}$ was proposed. For this method, colloid solutions of metallic copper particles were prepared by reducing copper ions derived from $\mathrm{CuCl}_{2}$ with hydrazine in the presence of citric acid and CTAB in water at a final copper concentration of $0.1 \mathrm{M}$. The particles had ca. submicrometer sizes, and many tiny particles with sizes of ca. $10-20 \mathrm{~nm}$, which were produced through the growth of particle nuclei generated at the final reaction stage by residual hydrazine, were deposited on the submicron-sized particles. The shear strength of the tiny particles/immobilized particles was as high as $41.7 \mathrm{MPa}$. This result could be explained by the melting points of the tiny particles being lower than those of the other large particles and the increased in bulk density via the dominant incorporation of tiny particles into the voids between the submicronsized particles.

Acknowledgements This work was partially supported by Hitachi, Ltd.

\section{Compliance with ethical standards}

Conflict of interest The authors declare no conflicts of interest.

\section{References}

1. Cheng S, Huang C-M, Pecht M (2017) A review of lead-free solders for electronics applications. Microelectron Reliab 75:77-95

2. Zhong Y, An R, Ma H, Wang C (2019) Low-temperature-solderable intermetallic nanoparticles for 3D printable flexible electronics. Acta Mater 162:163-175

3. Song G, Li T, Chen L (2018) The mechanical properties and interface bonding mechanism of immiscible $\mathrm{Mg} / \mathrm{steel}$ by laser-tungsten inert gas welding with filler wire. Mater Sci Eng A 736:306-315

4. Khatibi G, Kotas AB, Lederer M (2018) Effect of aging on mechanical properties of high temperature $\mathrm{Pb}$-rich solder joints. Microelectron Reliab 85:1-11

5. Weisman C (1976) Welding handbook, vol 1, 7th edn. American Welding Society, Miami

6. Zhu Y, Hou J, Yu G, Qiu J, Chen S, Zhou X (2016) Effects of exposing temperature on corrosion performance of weld joint of a $\mathrm{Ni}-\mathrm{Mo}-\mathrm{Cr}$ alloy. J Fluorine Chem 182:69-75

7. Ding Y, Wang J, Zhao M, Ju D (2018) Effect of annealing temperature on joints of diffusion bonded Mg/Al alloys. Trans Nonferrous Met Soc China 28:251-258

8. Khanzadeh M, Shiran G, Khalaj G, Pouraliakbar H, Jandaghi MR, Dehnavi AS, Bakhtiari H (2018) Multilayer Cu/Al/Cu explosive welded joints: characterizing heat treatment effect on the 
interface microstructure and mechanical properties. J Manuf Process 35:657-663

9. Tu K-N (2007) Solder joint technology. Springer, New York

10. Wang F, O'Keefe M, Brinkmeyer B (2009) Microstructural evolution and tensile properties of $\mathrm{Sn}-\mathrm{Ag}-\mathrm{Cu}$ mixed with $\mathrm{Sn}-\mathrm{Pb}$ solder alloys. J Alloys Compd 477:267-273

11. Wang F, Li D, Tian S, Zhang Z, Wang J, Yan C (2017) Interfacial behaviors of $\mathrm{Sn}-\mathrm{Pb}, \mathrm{Sn}-\mathrm{Ag}-\mathrm{Cu} \mathrm{Pb}$-free and mixed $\mathrm{Sn}-\mathrm{Ag}-\mathrm{Cu}$ / $\mathrm{Sn}-\mathrm{Pb}$ solder joints during electromigration. Microelectron Reliab 73:106-115

12. Li Q, Liu X, Lu S (2018) Corrosion behavior assessment of tin-lead and lead free solders exposed to fire smoke generated by burning polyvinyl chloride. Mater Chem Phys 212:298-307

13. Abtew M, Selvaduray $G$ (2000) Lead-free solders in microelectronics. Mater Sci Eng R 27:95-141

14. Noor EEM, Sharif NM, Yew CK, Ariga T, Ismail AB, Hussain Z (2010) Wettability and strength of $\mathrm{In}-\mathrm{Bi}-\mathrm{Sn}$ lead-free solder alloy on copper substrate. J Alloys Compd 507:290-296

15. Aamir M, Muhammad R, Ahmeda N, Waqas M (2017) Impact of thermal aging on the intermetallic compound particle size and mechanical properties of lead free solder for green electronics. Microelectron Reliab 78:311-318

16. El Basaty AB, Deghady AM, Eid EA (2017) Influence of small addition of antimony $(\mathrm{Sb})$ on thermal behavior, microstructural and tensile properties of $\mathrm{Sn}-9.0 \mathrm{Zn}-0.5 \mathrm{Al} \mathrm{Pb}$-free solder alloy. Mater Sci Eng A 701:245-253

17. Choi K, Yu D-Y, Ahn S, Kim K-H, Bang J-H, Ko Y-H (2018) Joint reliability of various $\mathrm{Pb}$-free solders under harsh vibration conditions for automotive electronics. Microelectron Reliab 86:66-71

18. Shibuta Y, Suzuki T (2010) Melting and solidification point of fcc-metal nanoparticles with respect to particle size: a molecular dynamics study. Chem Phys Lett 498:323-327

19. Loulijat H, Zerradi H, Mizani S, Achhal EM, Dezairi A, Ouaskit S (2015) The behavior of the thermal conductivity near the melting temperature of copper nanoparticle. J Mol Liq 211:695-704

20. Feng D, Feng Y, Yuan S, Zhang X, Wang G (2017) Melting behavior of Ag nanoparticles and their clusters. Appl Therm Eng 111:1457-1463

21. Ide E, Angata S, Hirose A, Kobayashi KF (2005) Metal-metal bonding process using Ag metallo-organic nanoparticles. Acta Mater 53:2385-2393

22. Bai JG, Zhang ZZ, Calata JN, Lu GQ (2006) Low-temperature sintered nanoscale silver as a novel semiconductor device-metallized substrate interconnect material. IEEE Trans Compon Pack Technol 29:589-593

23. Murray AJ, Jaroenapibal P, Koene B, Evoy S (2006) Sintering of silver nanoparticles for the formation of high temperature interconnect joints. Mater Res Soc Symp Proc 942:0942-W08-29

24. Yasuda Y, Ide E, Morita T (2009) Low-temperature bonding using silver nanoparticles stabilized by short-chain alkylamines. Jpn J Appl Phys 48:125004

25. Morisada Y, Nagaoka T, Fukusumi M, Kashiwagi Y, Yamamoto M, Nakamoto M (2010) A low-temperature bonding process using mixed Cu-Ag nanoparticles. J Electron Mater 39:1283-1288

26. Yan J, Zou G, Wu A, Ren J, Yan J, Hu A, Zhou Y (2012) Pressureless bonding process using Ag nanoparticle paste for flexible electronics packaging. Scripta Mater 66:582-585

27. Ishaka M, Maekawa K, Yamasaki K (2012) The characteristics of laser welded magnesium alloy using silver nanoparticles as insert material. Mater Sci Eng A 536:143-151

28. Eluri R, Paul BK (2012) Silver nanoparticle-assisted diffusion brazing of $3003 \mathrm{Al}$ alloy for microchannel applications. Mater Des 36:13-23

29. Ma C, Xue S, Bridges D, Palmer Z, Feng Z, Hu A (2017) Low temperature brazing nickel with Ag nanoparticle and $\mathrm{Cu}-\mathrm{Ag}$ coreshell nanowire nanopastes. J Alloys Compd 721:431-439
30. Kim K-S, Bang J-O, Jung S-B (2013) Electrochemical migration behavior of silver nanopaste screen-printed for flexible and printable electronics. Curr Appl Phys 13(S1):S190-S194

31. Lu G-Q, Yan C, Mei Y, Li X (2015) Dependence of electrochemical migration of sintered nanosilver on chloride. Mater Chem Phys 151:18-21

32. Yi P, Xiao K, Dong C, Zou S, Li X (2018) Effects of mould on electrochemical migration behaviour of immersion silver finished printed circuit board. Bioelectrochemistry 119:203-210

33. Tan CS, Lim DF, Ang XF, Wei J, Leong KC (2012) Low temperature $\mathrm{Cu}-\mathrm{Cu}$ thermo-compression bonding with temporary passivation of self-assembled monolayer and its bond strength enhancement. Microelectron Reliab 52:321-324

34. Ishizaki T, Miura D, Kuno A, Nagao R, Aoki S, Ohshima Y, Kino T, Usui M, Yamada Y (2016) Power cycle reliability of Cu nanoparticle joints with mismatched coefficients of thermal expansion. Microelectron Reliab 64:287-293

35. Nishikawa $\mathrm{H}$, Hirano T, Takemoto $T$, Terada N (2011) Effects of joining conditions on joint strength of $\mathrm{Cu} / \mathrm{Cu}$ joint using $\mathrm{Cu}$ nanoparticle paste. Open Surf Sci J 3:60-64

36. Yan J, Zou G, Wu A, Ren J, Hu A, Zhou YN (2012) Polymerprotected $\mathrm{Cu}-\mathrm{Ag}$ mixed NPs for low-temperature bonding application. J Electron Mater 41:1886-1892

37. Ishizaki T, Satoh T, Kuno A, Tane A, Yanase M, Osawa F, Yamada Y (2013) Thermal characterizations of Cu nanoparticle joints for power semiconductor devices. Microelectron Reliab 53:1543-1547

38. Mokhtari O, Nishikawa $\mathrm{H}$ (2016) The shear strength of transient liquid phase bonded $\mathrm{Sn}-\mathrm{Bi}$ solder joint with added $\mathrm{Cu}$ particles. Adv Powder Technol 27:1000-1005

39. Li J, Liang Q, Shi T, Fan J, Gong B, Feng C, Fan J, Liao G, Tang Z (2019) Design of Cu nanoaggregates composed of ultra-small $\mathrm{Cu}$ nanoparticles for $\mathrm{Cu}-\mathrm{Cu}$ thermocompression bonding. J Alloys Compd 772:793-800

40. Krasovskii PV, Samokhin AV, Fadeev AA, Alexeev NV (2016) Thermal evolution study of nonmetallic impurities and surface passivation of Cu nanopowders produced via a DC thermal plasma synthesis. Adv Powder Technol 27:1669-1676

41. Mousavi-Kamazani M, Zarghami Z, Rahmatolahzadeh R, Ramezani M (2017) Solvent-free synthesis of $\mathrm{Cu}-\mathrm{Cu}_{2} \mathrm{O}$ nanocomposites via green thermal decomposition route using novel precursor and investigation of its photocatalytic activity. Adv Powder Technol 28:2078-2086

42. Lisiecki I, Billoudet F, Pileni MP (1996) Control of the shape and the size of copper metallic particles. J Phys Chem 100:4160-4166

43. Huang HH, Yan FQ, Kek YM, Chew CH, Xu GQ, Ji W, Oh PS, Tang SH (1997) Synthesis, characterization, and nonlinear optical properties of copper nanoparticles. Langmuir 1:172-175

44. Wu S-H, Chen D-H (2004) Synthesis of high-concentration Cu nanoparticles in aqueous CTAB solutions. J Colloid Interface Sci 273:165-169

45. Zhang X, Yin H, Cheng X, Hu H, Yu Q, Wang A (2006) Effects of various polyoxyethylene sorbitan monooils (Tweens) and sodium dodecyl sulfate on reflux synthesis of copper nanoparticles. Mater Res Bull 41:2041-2048

46. Khanna PK, Gaikwad S, Adhyapak PV, Singh N, Marimuthu R (2007) Synthesis and characterization of copper nanoparticles. Mater Lett 61:4711-4714

47. Yokoyama S, Takahashi H, Itoh T, Motomiya K, Tohji K (2014) Synthesis of metallic Cu nanoparticles by controlling Cu complexes in aqueous solution. Adv Powder Technol 25:999-1006

48. Kobayashi Y, Shirochi T, Yasuda Y, Morita T (2011) Preparation of metallic copper nanoparticles in aqueous solution and their bonding properties. Solid State Sci 13:553-558 
49. Kobayashi Y, Shirochi T, Yasuda Y, Morita T (2012) Metal-metal bonding process using metallic copper nanoparticles prepared in aqueous solution. Int J Adhes Adhes 33:50-55

50. Kobayashi Y, Shirochi T, Yasuda Y, Morita T (2013) Preparation of metallic copper nanoparticles by reduction of copper ions in aqueous solution and their metal-metal bonding properties. Int J Phys Nat Sci Eng 7:150-153

51. Kobayashi Y, Shirochi T, Maeda T, Yasuda Y, Morita T (2013) Microstructure of metallic copper nanoparticles/metallic disc interface in metal-metal bonding using them. Surf Interface Anal 45:1424-1428

52. Maeda T, Kobayashi Y, Yasuda Y, Morita T (2014) Metal-metal bonding properties of copper oxide nanoparticles. e-J Surf Sci Nanotechnol 12:105-108

53. Kobayashi Y, Abe Y, Maeda T, Yasuda Y, Morita T (2014) A metalmetal bonding process using metallic copper nanoparticles produced by reduction of copper oxide nanoparticles. J Mater Res Technol 3:114-121

54. Maeda T, Nakazawa H, Kobayashi Y, Yasuda Y, Morita T (2014) Effects of reductant concentration and reduction temperature in synthesis of copper nanoparticles on their metal-metal bonding properties. Sci Lett 8:1-9

55. Kobayashi Y, Yasuda Y, Morita T (2016) Recent advances in the synthesis of copper-based nanoparticles for metal-metal bonding processes. J Sci Adv Mater Dev 1:413-430
56. Kobayashi Y, Nakazawa H, Maeda T, Yasuda Y, Morita T (2017) Synthesis of metallic copper nanoparticles and metal-metal bonding process using them. Adv Nano Res 5:359-372

57. Morita T, Ide E, Yasuda Y, Hirose A (2008) Study of bonding technology using silver nanoparticles. Jpn J Appl Phys 47:6615-6622

58. Morita T, Yasuda Y, Ide E, Akada Y, Hirose A (2008) Bonding technique using micro-scaled silver-oxide particles for in-situ formation of silver nanoparticles. Mater Trans 49:2875-2880

59. Morita T, Yasuda Y, Ide E, Hirose A (2009) Direct bonding to aluminum with silver-oxide microparticles. Mater Trans 50:226-228

60. Li Z, Li J, Xu R, Hong Z, Liu Z (2015) Streaming potential method for characterizing the overlapping of diffuse layers of the electrical double layers between oppositely charged particles. Colloids Surf A 478:22-29

61. Dimic-Misic K, Hummel M, Paltakari J, Sixta H, Maloney T, Gane $P$ (2015) From colloidal spheres to nanofibrils: extensional flow properties of mineral pigment and mixtures with micro and nanofibrils under progressive double layer suppression. J Colloid Interface Sci 446:31-43

62. Lisuzzo L, Cavallaro G, Parisi F, Milioto S, Lazzara G (2019) Colloidal stability of halloysite clay nanotubes. Ceram Int 45:2858-2865

Publisher's Note Springer Nature remains neutral with regard to jurisdictional claims in published maps and institutional affiliations. 